\title{
DICOM-RT Plan Complexity Verification for Volumetric Modulated Arc Therapy
}

\author{
Hideharu Miura ${ }^{1}$, Masao Tanooka ${ }^{2}$, Hiroyuki Inoue ${ }^{2}$, Masayuki Fujiwara ${ }^{1}$, Kengo Kosaka ${ }^{1}$, \\ Hiroshi Doi', Yasuhiro Takada1, Soichi Odawara', Norihiko Kamikonya', Shozo Hirota1 \\ ${ }^{1}$ Department of Radiology, Hyogo College of Medicine, Nishinomiya City, Japan \\ ${ }^{2}$ Department of Radiological Technology, Hyogo College of Medicine College Hospital, Nishinomiya City, Japan \\ Email: ${ }^{*}$ hide-miura@osaka-igrt.or.jp
}

Received 9 April 2014; revised 8 May 2014; accepted 5 June 2014

Copyright (C) 2014 by authors and Scientific Research Publishing Inc.

This work is licensed under the Creative Commons Attribution International License (CC BY).

http://creativecommons.org/licenses/by/4.0/

(c) (i) Open Access

\begin{abstract}
The purpose of this study was to investigate the relationship between plan parameters verified with DICOM-RT and dosimetric results for volumetric modulated arc therapy (VMAT). We investigated three treatment locations: prostate cancer (ten cases), maxillary sinus cancer (four cases), and malignant pleura mesothelioma (four cases) with treatment plans generated by a Monaco ${ }^{\mathrm{TM}}$ treatment planning system (TPS), and delivered with an Elekta Synergy ${ }^{\mathrm{TM}}$ linear accelerator. We calculated plan parameters, including gantry and multileaf collimator (MLC) positions, Monitor Units (MU), and millimeters of MLC motion per degree of gantry rotation (mm/degree), and performed quality assurance (QA) with a DICOM-RT plan verification system. We measured the VMAT dose with a two-dimensional diode array detector. The average gamma passing rate with percent dose acceptance criteria and distance to agreement criteria of $2 \mathrm{~mm}$ and $2 \%(2 \mathrm{~mm} / 2 \%)$ were $\mathbf{9 7 . 4 \%}, \mathbf{9 7 . 8 \%}$ and $\mathbf{9 2 . 0} \%$ for prostate cancer, maxillary sinus cancer, and malignant pleural mesothelioma, respectively. The mean 95th percentile value for DICOM-calculated $\mathrm{mm} /$ degree was 4.0, 5.2, and 11.1 for prostate cancer, maxillary sinus cancer, and malignant pleural mesothelioma, respectively. The gamma passing rate showed a correlation with calculated $\mathrm{mm} / \mathrm{degree}$, with a coefficient of determination $\left(R^{2}\right)$ of 0.60 . Higher calculated $\mathrm{mm} /$ degree values led to increased dosimetric errors. We conclude that dose distribution calculated by a TPS is more reliable at smaller $\mathrm{mm} /$ degree.
\end{abstract}

\section{Keywords}

Volumetric Modulated Arc Therapy, Quality Assurance, Radiation Treatment Planning, Radiation Dosimetry

\footnotetext{
${ }^{*}$ Corresponding author.
}

How to cite this paper: Miura, H., et al. (2014) DICOM-RT Plan Complexity Verification for Volumetric Modulated Arc Therapy. International Journal of Medical Physics, Clinical Engineering and Radiation Oncology, 3, 117-124. 


\section{Introduction}

Volumetric modulated arc therapy (VMAT) is a new radiotherapy technique which allows the generation of treatment plans of similar or better quality than those for fixed-field intensity modulated radiation therapy (IMRT) while reducing treatment time per fraction [1].

Patient-specific quality assurance (QA) for VMAT plans is important in confirming dose distribution. Dosimetric verification is mostly often performed by delivering the treatment plan to a solid water phantom, measuring dosage with an ionization chamber and radiochromic film, and comparing the measured dose to the calculated dose [2]. Various QA devices for VMAT delivery verification have recently been introduced, including a cylindrical diode array (ArcCHECK ${ }^{\mathrm{TM}}$, Sun Nuclear Corporation, Melbourne, FL, USA), two orthogonal diode arrays (Delta4 ${ }^{\mathrm{TM}}$ phantom, Scandidos, Uppsala, Sweden), an octagonal phantom (Octavius ${ }^{\mathrm{TM}}$, PTW, Freiburg, Germany), and a two-dimensional ionization chamber array with a gantry-mounted detector matrix to measure fluence (MatriXX/COMPASS ${ }^{\mathrm{TM}}$, IBA Dosimetry, Schwarzenbruck, Germany) [3]-[5]. However, these methods do not evaluate other plan parameters, such as gantry angle or multileaf collimator (MLC) position during VMAT delivery. Rather, we consider it important to analyze plan parameters that influence dosimetric accuracy.

Several authors have reported methods for assessing IMRT and VMAT plan complexity [6]-[12]. The complexity of IMRT and VMAT plans leads to a decreased gamma passing rate in dosimetric results [6] [7] [11]. Our previous study investigated dosimetry measurements of VMAT plan parameters using DICOM-RT verification and compared them to mechanical dose errors using a log file [13]. Despite the necessity of assessing VMAT plan complexity to ensure secure treatment planning, however, methods for quantitative assessment of VMAT plan complexity are still lacking.

The purpose of this study was to compare DICOM-RT plan parameters with VMAT dose measured with a 2D array detector.

\section{Materials and Methods}

\subsection{Treatment Planning}

We randomly selected ten prostate cancer, four maxillary sinus cancer, and four malignant pleural mesothelioma cases and created VMAT plans. These patients underwent computed tomography scanning (Aquilion LB ${ }^{\mathrm{TM}}$, Toshiba, Otawara, Japan) of the area to be treated. Prostate cancer patients also underwent magnetic resonance imaging (MRI). Monte Carlo dose calculations (Monaco ${ }^{\mathrm{TM}}$ version 3.0, Elekta, Maryland Heights Missouri, USA) were performed on the CT data with a grid size of $3 \mathrm{~mm}$ and variance of $3 \%$. A linear accelerator (Synergy ${ }^{\mathrm{TM}}$, Elekta, Crawley, UK) with a 6 or $10 \mathrm{MV}$ photon beam and an MLC leaf width of 10 mm was used for VMAT delivery. Treatment plans were transferred from the TPS to a Desktop Pro $7.01^{\mathrm{TM}}$ linac control system via a record and verify system (Mosaiq ${ }^{\mathrm{TM}}$ version 1.6, Elekta, Sunnyvale, CA, USA).

\subsubsection{Prostate Cancer}

Gross tumor volume (GTV) was defined as visible tumor on CT and MRI. The clinical target volume (CTV) was defined as the prostate plus the proximal half of the seminal vesicles. The planning target volume (PTV) was formed by extending the CTV $10 \mathrm{~mm}$ outward in the axial plane except posteriorly in the region of the rectum-prostate interface, where the margin was set at $5 \mathrm{~mm}$. The rectum, bladder, and femoral head were contoured as organs at risk (OAR). All plans consisted of a $360^{\circ}$ clockwise arc with a $0^{\circ}$ collimator angle. A dose of 74 Gy in 37 fractions was prescribed to 95\% of the PTV, and maximum dose was restricted to $110 \%$ of the prescribed dose. Dose constraints for OARs were as follows: percentage of volume receiving 70 Gy (V70Gy) < 15\%; V60Gy < 25\%; V40 Gy < 50\%; bladder, V65Gy < 25\%, V40Gy < 50\%. The range of PTVs was 53.7 117.4 cc. The range of Monitor Units (MU) was 415 - 612.

\subsubsection{Maxillary Sinus Cancer}

GTV was defined as visible tumor at the brain window settings. CTV was defined by adding an isotropic margin of $5 \mathrm{~mm}$ to the GTV. PTV was defined by adding an isotropic margin of $3 \mathrm{~mm}$ to the CTV. The normal brain, brainstem, spinal cord, optic chiasm, ipsilateral and contralateral eyes, and ipsilateral and contralateral optic nerves were contoured as OARs. A dose of 66 Gy in 33 fractions was prescribed to 95\% of the PTV, and maxi- 
mum dose was restricted to $110 \%$ of the prescribed dose. The dose-volume criteria used for the VMAT optimization can be found in our previous work [14]. We used gantry rotation angles of $180^{\circ}$. The range of PTVs was 68.6 - 175.4 cc. The range of MU was 342 - 464.

\subsubsection{Malignant Pleural Mesothelioma}

The CTV was defined as the entire hemithoracic pleural space and involved nodal stations. The PTV was generally extended by $5 \mathrm{~mm}$ outside the CTV. The contralateral lung, liver, heart, esophagus, kidneys, and spinal cord were contoured as OARs. A dose of $54 \mathrm{~Gy}$ in 30 fractions was prescribed to $95 \%$ of the PTV, and maximum dose was restricted to $115 \%$ of the prescribed dose. Dose constraints for OARs were given as follows: contralateral lung, V5Gy < 60\%, V20Gy < 10\%, mean dose < 10.0 Gy; liver, V30Gy < 33\%, mean dose < 30 Gy; heart, V45Gy < 50\%, D1\% < 60 Gy; contralateral kidney, V15Gy < 20\%; and spinal cord, Dmax < 47 Gy. We used two gantry rotation angles of $210^{\circ}$ to reduce dose to the contralateral lung. The first arc rotation was clockwise, and the second arc was counterclockwise. The range of PTVs was 1346.7 - 2920.2 cc. The range of MU was 634 - 994.

\subsection{Calculation of Plan Parameters}

We calculated plan parameters using MATLAB software (MathWorks Inc., Natick, MA, USA), defining the specified gantry angle position, MLC leaf position, and cumulative delivered MUs for each segment. We calculated the gantry angle position, MLC positions, and MUs with a DICOM-RT plan which calculated the gantry and MLC parameters for each segment. We defined the gantry parameters from differences in position between gantry angle positions for each segment (degrees/MU). We defined MLC parameters using the number of MU at different positions of adjacent MLC leaves for each segment (mm/MU). We defined comprehensive parameters from positions of adjacent MLC leaves between gantry angle positions for each segment (mm/degree). The absolute value of the 95th percentile of the plan parameter for VMAT was investigated.

\subsection{Dosimetric Measurement}

The dosimetric QA system used for dose verification measurements of the VMAT delivery consisted of a twodimensional multiple-diode array detector (MapCHECK2 ${ }^{\mathrm{TM}}$, Sun Nuclear Corporation, Melbourne, FL, USA) encased within a rectangular solid water phantom (MapPHAN2 ${ }^{\mathrm{TM}}$, Sun Nuclear Corporation, Melbourne, FL, USA). The enclosure phantom has a cross-sectional area of $30 \times 30 \mathrm{~cm}^{2}$ parallel to the detector plane. Because the detector's build-up material was not made of water equivalent, the depth from the phantom surface to the detector plane was adjusted to be a water-equivalent length of $10 \mathrm{~cm}$ on each side of the detector plane. Total dose distribution during VMAT delivery was measured in the coronal plane by the detector. The source-to-detector distance was $100 \mathrm{~cm}$ when the beam was delivered perpendicular to the detector plane. Gamma analysis with percent dose acceptance criteria and distance to agreement criteria of $3 \% / 3 \mathrm{~mm}$ and $2 \% / 2 \mathrm{~mm}(3 \% / 3 \mathrm{~mm}$, $2 \% / 2 \mathrm{~mm}$ ) passing criteria under a dose threshold of $10 \%$ of the maximum dose on each plane were used. The Van Dyk percent difference criterion was used [15].

\section{Results}

Table 1 shows the gamma passing rate analysis of the dose calculated vs. measured by the detector. The gamma passing rate $(3 \% / 3 \mathrm{~mm})$ of all treatment sites was approximately $100 \%$ for the VMAT plan. Gamma passing rates using stricter gamma criteria $(2 \% / 2 \mathrm{~mm}$ ) were $97.4 \%$ (range: $93.8 \%-100.0 \%)$, $97.8 \%$ (range: $94.3 \%$ 99.4\%) and $92.0 \%$ (range: $89.3 \%$ - 94.6\%) for prostate cancer, maxillary sinus cancer, and malignant pleural mesothelioma VMAT deliveries, respectively. Malignant pleural mesothelioma showed lower passing rates than prostate and maxillary sinus cancers.

Figures 1-3 show frequency histograms of calculated parameters for prostate cancer, maxillary sinus cancer, and malignant pleural mesothelioma VMAT plans, respectively. Averages of 95th-percentile values for the calculated plan parameters for the three treatment sites are summarized in Table 2. Calculated degrees/MU for malignant pleural mesothelioma VMAT plans were lower than those for the other treatment sites. In contrast, calculated $\mathrm{mm} / \mathrm{MU}$ and $\mathrm{mm} /$ degree for malignant pleural mesothelioma VMAT plans were higher than those for the other treatment sites. 
Figures 4-6 show plots of calculated degrees/MU, $\mathrm{mm} / \mathrm{MU}$, and $\mathrm{mm} /$ degree as a function of the gamma passing rate $(2 \% / 2 \mathrm{~mm})$ for all cases. The gamma passing rate did not correlate with calculated degrees/MU or mm/MU, with a coefficient of determination $\left(\mathrm{R}^{2}\right)$ of 0.27 and 0.39 , respectively. Conversely, the gamma passing rate correlated with calculated $\mathrm{mm} /$ degree, with a coefficient of determination $\left(\mathrm{R}^{2}\right)$ of 0.60 .

\section{Discussions}

In this study, we investigated the relationship between plan parameters and dosimetric results for VMAT delivery. The dosimetric results $(3 \% / 3 \mathrm{~mm})$ showed VMAT was capable of delivering accurate doses for all treatment

Table 1. Gamma analysis passing rates measured by 2D diode detector. Percent dose acceptance criteria and distance to agreement criteria are $(3 \% / 3 \mathrm{~mm})$ and $(2 \% / 2 \mathrm{~mm})$ for clinical criteria and more restricted constraints, respectively. The analyzed data are displayed as mean with ranges in parentheses.

\begin{tabular}{ccc}
\hline & $3 \mathrm{~mm} / 3 \%$ & $2 \mathrm{~mm} / 2 \%$ \\
\hline Prostate cancer & $99.9 \%(99.7-100.0)$ & $97.4 \%(93.8-100.0)$ \\
Maxillary sinus cancer & $99.9 \%(99.6-100.0)$ & $97.8 \%(94.3-99.4)$ \\
Malignant pleural mesothelioma & $99.2 \%(98.5-99.0)$ & $92.0 \%(89.3-94.6)$ \\
\hline
\end{tabular}

Table 2. Mean 95th-percentile values for DICOM-calculated plan parameters, with range in parentheses.

\begin{tabular}{cccc}
\hline & degrees/MU & $\mathrm{mm} / \mathrm{MU}$ & $\mathrm{mm} / \mathrm{degree}$ \\
\hline Prostate cancer & $5.4(2.5-7.9)$ & $12.2(6.3-18.8)$ & $4.1(3.2-4.2)$ \\
Maxillary sinus cancer & $3.1(1.7-5.1)$ & $10.0(5.2-18.5)$ & $5.2(4.3-6.3)$ \\
Malignant pleural mesothelioma & $2.8(2.1-3.3)$ & $17.8(9.3-21.8)$ & $11.1(10.2-11.6)$ \\
\hline
\end{tabular}

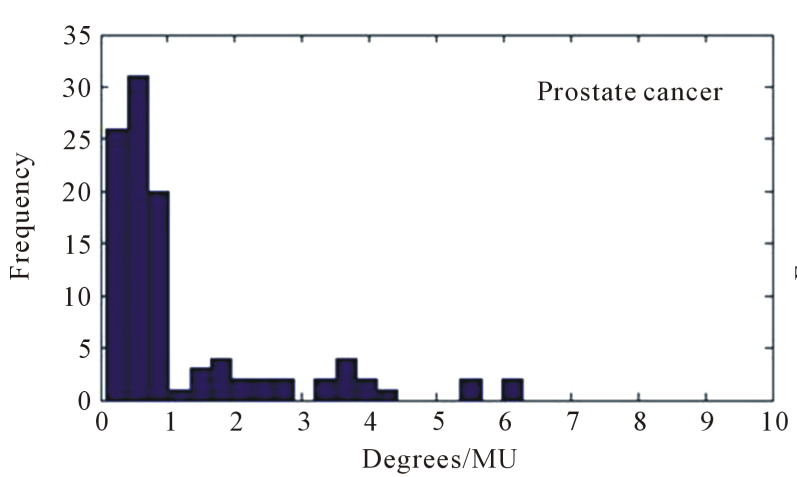

(a)

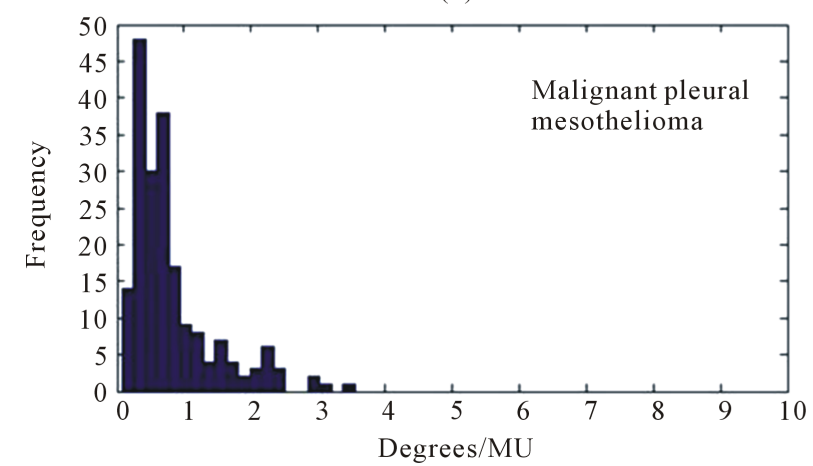

(c)

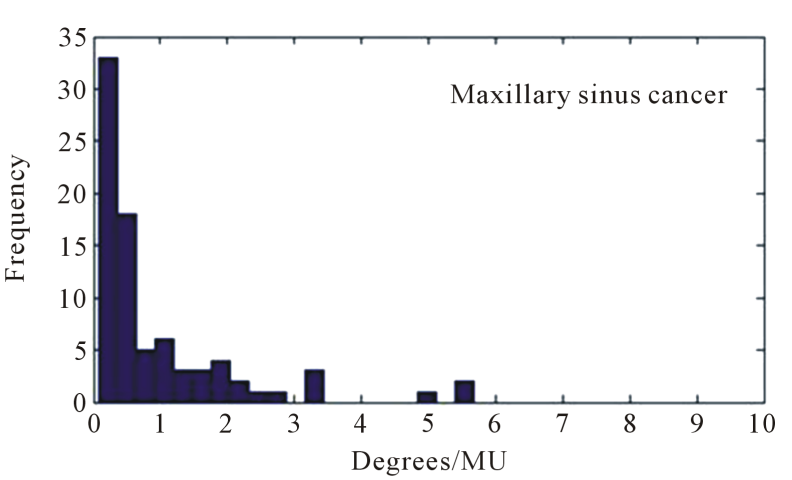

(b)

Figure 1. Frequency histograms of calculated degrees/MU for (a) prostate cancer, (b) maxillary sinus cancer, and (c) malignant pleural mesothelioma VMAT plans, respectively. 


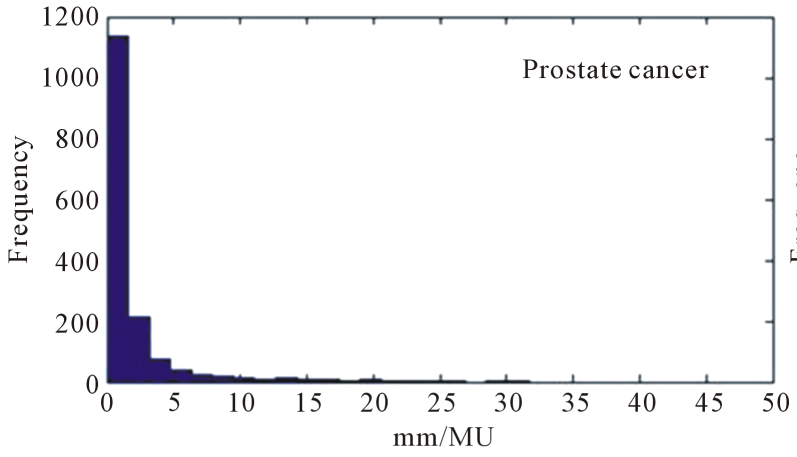

(a)

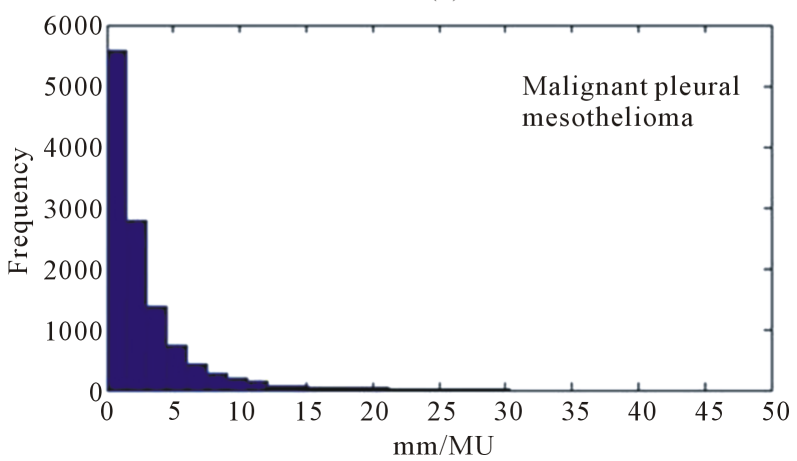

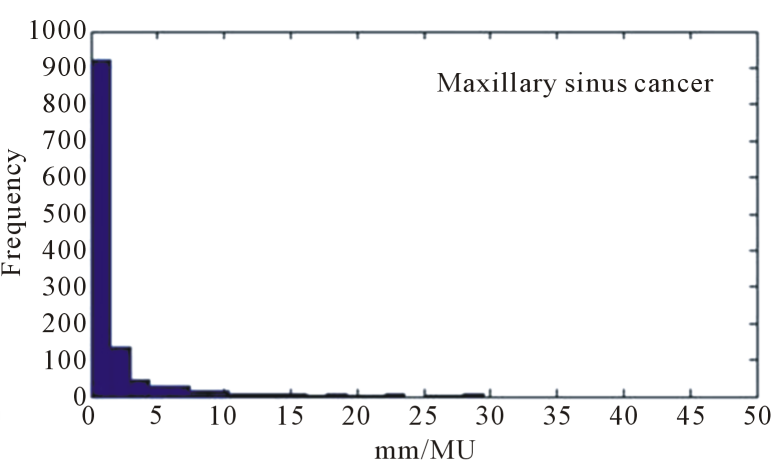

(b)

(c)

Figure 2. Frequency histograms of calculated mm/MU for (a) prostate cancer, (b) maxillary sinus cancer, and (c) malignant pleural mesothelioma VMAT plans, respectively.

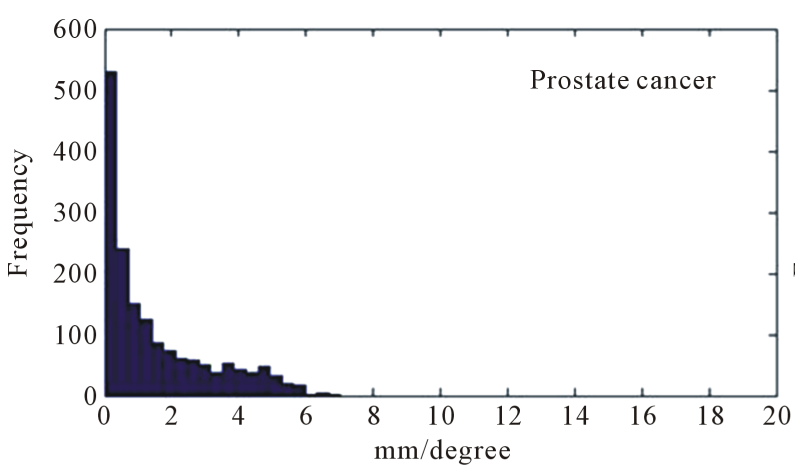

(a)

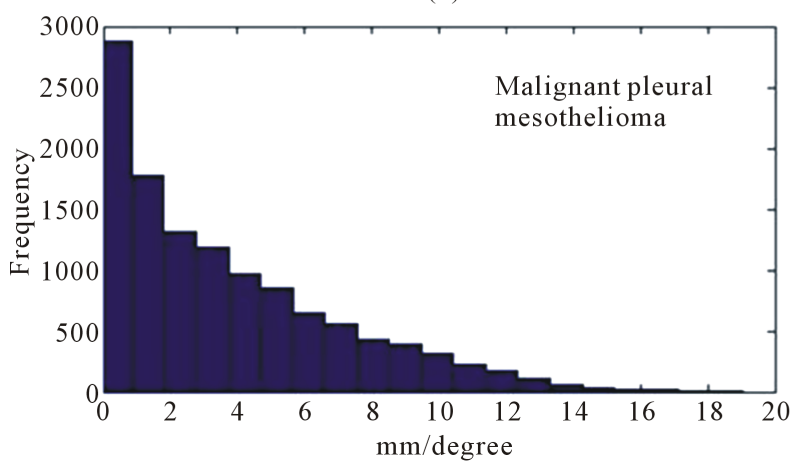

(c)

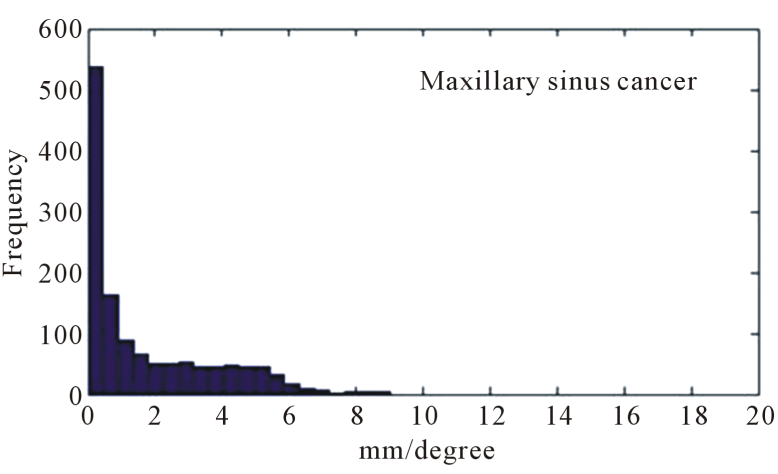

(b)

Figure 3. Frequency histograms of calculated mm/degree for (a) prostate cancer, (b) maxillary sinus cancer, and (c) malignant pleural mesothelioma VMAT plans, respectively. 


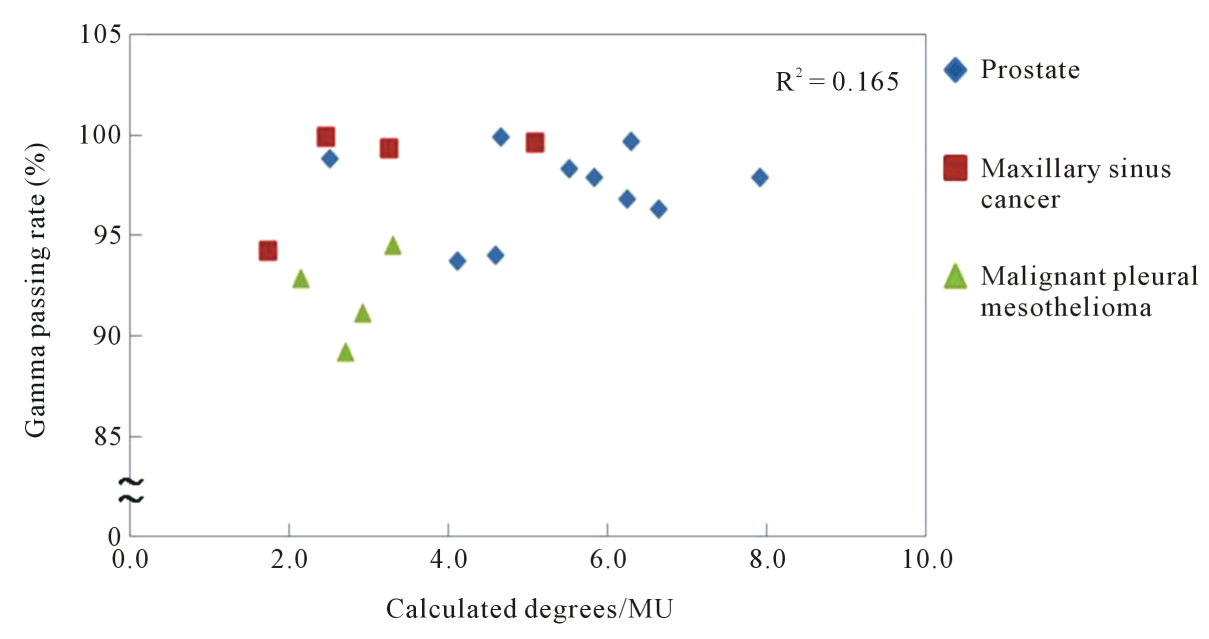

Figure 4. Gamma passing rate as a function of gantry angle divided by MU for each segment (degrees/MU). Gamma passing rate did not correlate with calculated degrees/MU.

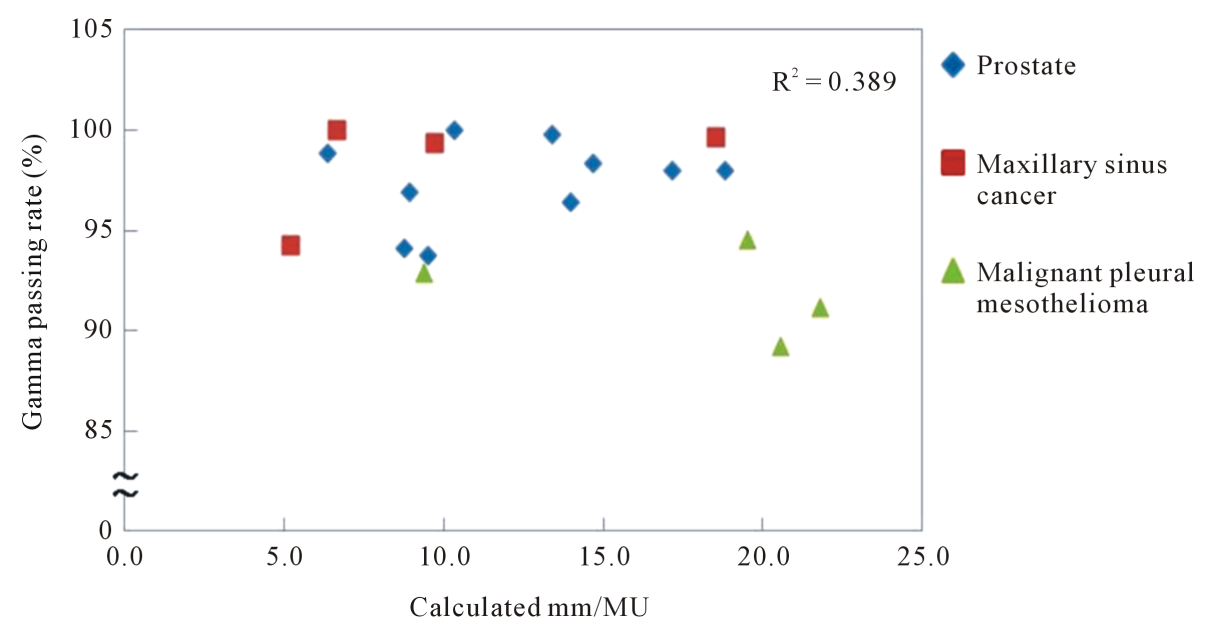

Figure 5. Gamma passing rate as a function of MLC travel distance divided by MU for each segment (mm/MU). Gamma passing rate did not correlate with calculated mm/MU.

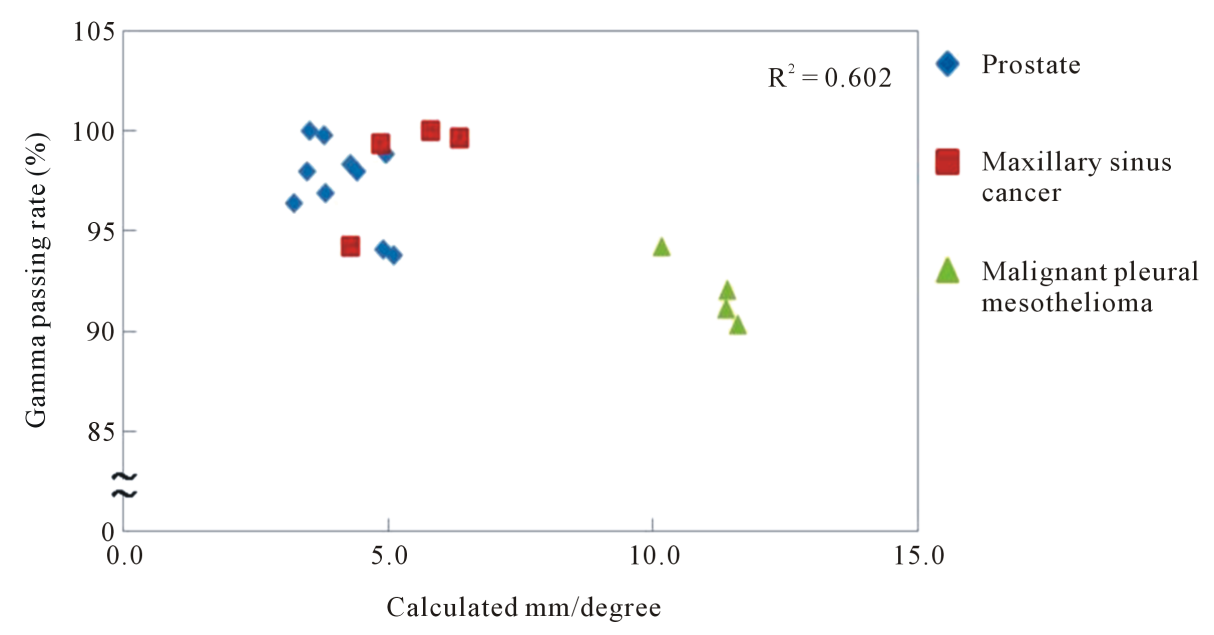

Figure 6. Gamma passing rate as a function of MLC travel distance divided by gantry angle for each segment (mm/degree). Gamma passing rates correlated with calculated mm/degree. 
sites offered to patients. In our clinical practice, the tolerance level for passing rates is set to $95 \%$ at $3 \%$ and 3 $\mathrm{mm}$ gamma analysis. A $95 \%$ pass rate at $3 \%$ and $3 \mathrm{~mm}$ was obtained in $100 \%$ of the evaluated plans. This is clearly a good result which confirms the reliability of VMAT planning and delivery. The average gamma passing rates $(2 \% / 2 \mathrm{~mm})$ were $97.4 \%, 97.8 \%$, and $92.0 \%$ for prostate cancer, maxillary sinus cancer, and malignant pleural mesothelioma VMAT deliveries, respectively. Malignant pleural mesothelioma VMAT delivery offered a slightly lower passing rate in the gamma index analysis. This finding is useful in understanding the beam characteristics of VMAT delivery. Limitations of our experimental devices include a lack of dosimetric accuracy for film dosimetry and spatial resolution issues for 2D diode arrays. Given the complexity of VMAT delivery, an accurate high resolution 3D quality assurance system would be ideal.

Plan parameters for VMAT differed for the three treatment sites. Calculated $\mathrm{mm} / \mathrm{MU}$ and $\mathrm{mm} /$ degree for malignant pleural mesothelioma VMAT deliveries were higher than for other treatment sites, because malignant pleural mesothelioma is associated with a much larger PTV. Accordingly, degrees/MU for malignant pleural mesothelioma VMAT delivery were lower than those for prostate cancer and maxillary sinus cancer patients, because malignant pleural mesothelioma requires a larger total MU. Total MU can be easily used to determine beam complexity, but cannot be used to assess VMAT plan complexity, because beam path-length differs by patient and treatment site. Other indices for evaluating VMAT plan complexity are available. Masi et al. reported that average leaf travel (LT), modulation complexity score applied to VMAT (MCSv), MU value, and a multiplicative combination of LT and MCSv (LTMCS) were potential indices for scoring VMAT plan complexity using threshold values [11].

The gamma passing rate showed a relationship with calculated $\mathrm{mm} /$ degree. The complexity of the VMAT plan is mainly due to the need to account for the motion of the gantry and MLC during rotation. Plan complexity is also affected by PTV size, locations of the tumor and OARs, required tumor dose, and dose-volume constraints of the OAR. Additionally, VMAT delivery also differs from IMRT delivery with regard to MLC motion. MLC motion in VMAT delivery is back and forth, resulting in a large amount of leaf travel; this movement may lead to discrepancies between calculated and delivered doses. Larger PTVs may need special attention to the increased mm/degree with MLC travel (Figure 7). Chen et al. reported that the average gamma passing rate decreased from $98 \%$ to $80 \%$ when the allowable leaf motion increased from 3 to $20 \mathrm{~mm} /$ degree [16]. If such motion were used to achieve a VMAT plan, control point angular separation would need to be more precise [11] [16]. Calculated $\mathrm{mm} /$ degree can be easily computed and understood.

\section{Conclusion}

We showed a relationship between DICOM-RT plan parameters and passing rates obtained by pretreatment dosimetric verification plans. DICOM-calculated $\mathrm{mm}$ /degree can be used to assess VMAT plan complexity, although increasing $\mathrm{mm} /$ degree may lead to increased dosimetric errors. We conclude that dose distribution calculated by

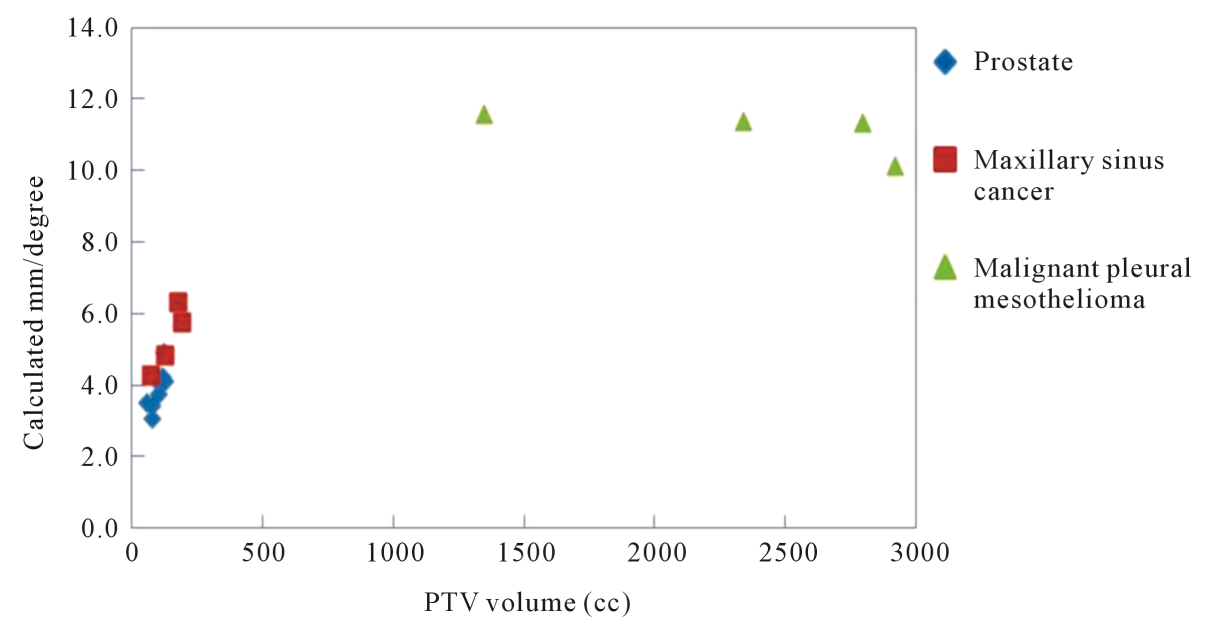

Figure 7. Calculated $\mathrm{mm}$ /degree as a function of PTV volume. Larger PTV volume led to a higher calculated $\mathrm{mm} /$ degree, resulting from a greater distance of leaf travel. 
a TPS is more reliable at smaller mm/degree.

\section{References}

[1] Otto, K. (2008) Volumetric Modulated Arc Therapy: IMRT in a Single Gantry Arc. Medical Physics, 35, 310-317. http://dx.doi.org/10.1118/1.2818738

[2] Bedford, J.L. and Warrington, A.P. (2009) Commissioning of Volumetric Modulated Arc Therapy (VMAT). International Journal of Radiation Oncology* Biology*Physics, 73, 537-545. http://dx.doi.org/10.1016/j.ijrobp.2008.08.055

[3] Létourneau, D., Publicover, J., Kozelka, J., Moseley, D.J. and Jaffray, D.A. (2009) Novel Dosimetric Phantom for Quality Assurance of Volumetric Modulated Arc Therapy. Medical Physics, 36, 1813-1821. http://dx.doi.org/10.1118/1.3117563

[4] Bedford, J.L., Lee, Y.K., Wai, P., South, C.P. and Warrington, A.P. (2009) Evaluation of the Delta4 Phantom for IMRT and VMAT Verification. Physics in Medicine and Biology, 54, N167-N176. http://dx.doi.org/10.1088/0031-9155/54/9/N04

[5] Boggula, R., Birkner, M., Lohr, F., Steil, V., Wenz, F. and Wertz, H. (2011) Evaluation of a 2D Detector Array for PatientSpecific VMAT QA with Different Setups. Physics in Medicine and Biology, 56, 7163-7177. http://dx.doi.org/10.1088/0031-9155/56/22/011

[6] Younge, K.C., Matuszak, M.M., Moran, J.M., McShan, D.L., Fraass, B.A. and Roberts, D.A. (2012) Penalization of Aperture Complexity in Inversely Planned Volumetric Modulated Arc Therapy. Medical Physics, 39, 7160-7170. http://dx.doi.org/10.1118/1.4762566

[7] McGarry, C.K., Chinneck, C.D., O’Toole, M.M., O’Sullivan, J.M., Prise, K.M. and Hounsell, A.R. (2011) Assessing Software Upgrades, Plan Properties and Patient Geometry Using Intensity Modulated Radiation Therapy (IMRT) Complexity Metrics. Medical Physics, 38, 2027-2034. http://dx.doi.org/10.1118/1.4762566

[8] Webb, S. (2003) Use of a Quantitative Index of Beam Modulation to Characterize Dose Conformality: Illustration by a Comparison of Full Beamlet IMRT, Few-Segment IMRT (fsIMRT) and Conformal Unmodulated Radiotherapy. Physics in Medicine and Biology, 48, 2051-2062. http://dx.doi.org/10.1088/0031-9155/48/14/301

[9] Mohan, R., Arnfield, M., Tong, S., Wu, Q. and Siebers, J. (2000) The Impact of Fluctuations in Intensity Patterns on the Number of Monitor Units and the Quality and Accuracy of Intensity Modulated Radiotherapy. Medical Physics, 27, 1226-1037. http://dx.doi.org/10.1118/1.599000

[10] McNiven, A.L., Sharpe, M.B. and Purdie, T.G. (2010) A New Metric for Assessing IMRT Modulation Complexity and Plan Deliverability. Medical Physics, 37, 505-515. http://dx.doi.org/10.1118/1.3276775

[11] Masi, L., Doro, R., Favuzza, V., Cipressi, S. and Livi, L. (2013) Impact of Plan Parameters on the Dosimetric Accuracy of Volumetric Modulated Arc Therapy. Medical Physics, 40, Article ID: 071718. http://dx.doi.org/10.1118/1.4810969

[12] Du, W., Cho, S.H., Zhang, X., Hoffman, K.E. and Kudchadker, R.J. (2014) Quantification of Beam Complexity in Intensity-Modulated Radiation Therapy Treatment Plans. Medical Physics, 41, Article ID: 021716. http://dx.doi.org/10.1118/1.4861821

[13] Miura, H., Tanooka, M., Fujiwara, M., et al. (2014) Predicting Delivery Error Using a DICOM-RT Plan for Volumetric Modulated Arc Therapy. IJMPCERO, 3, 98-106. http://www.scirp.org/journal/PaperInformation.aspx?PaperID=45720

[14] Miura, H., Fujiwara, M., Tanooka, M., et al. (2012) Dosimetric and Delivery Characterizations of Full-Arc and Half-Arc Volumetric-Modulated Arc Therapy for Maxillary Cancer. Journal of Radiation Research, 53, 785-790. http://dx.doi.org/10.1093/jrr/rrs03

[15] Van Dyk, J., Barnett, R.B., Cygler, J.E. and Schragge, P.C. (1993) Commissioning and Quality Assurance of Treatment Planning Computers. International Journal of Radiation Oncology*Biology*Physics, 26, 261-273. http://dx.doi.org/10.1016/0360-3016(93)90206-B

[16] Chen, F., Rao, M., Ye, J.S., Shepard, D.M. and Cao, D. (2011) Impact of Leaf Motion Constraints on IMAT Plan Quality, Delivery Accuracy, and Efficiency. Medical Physics, 38, 6106-6118. http://dx.doi.org/10.1118/1.3651698 
Scientific Research Publishing (SCIRP) is one of the largest Open Access journal publishers. It is currently publishing more than 200 open access, online, peer-reviewed journals covering a wide range of academic disciplines. SCIRP serves the worldwide academic communities and contributes to the progress and application of science with its publication.

Other selected journals from SCIRP are listed as below. Submit your manuscript to us via either submit@scirp.org or Online Submission Portal.
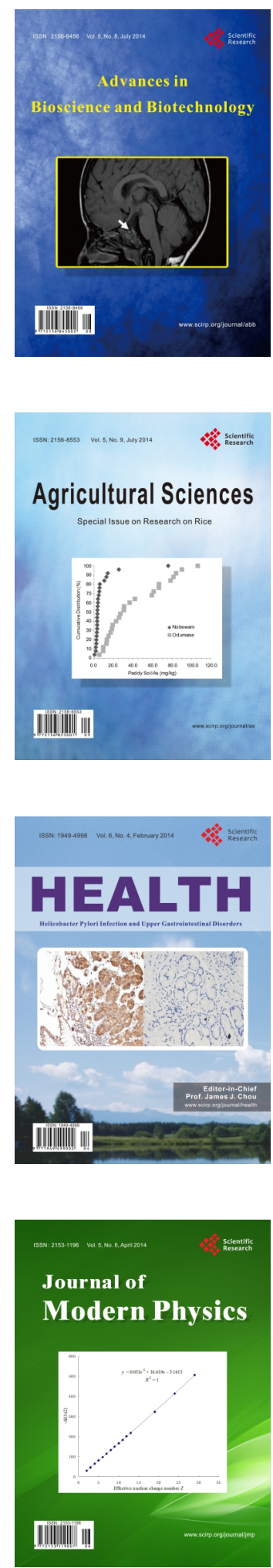
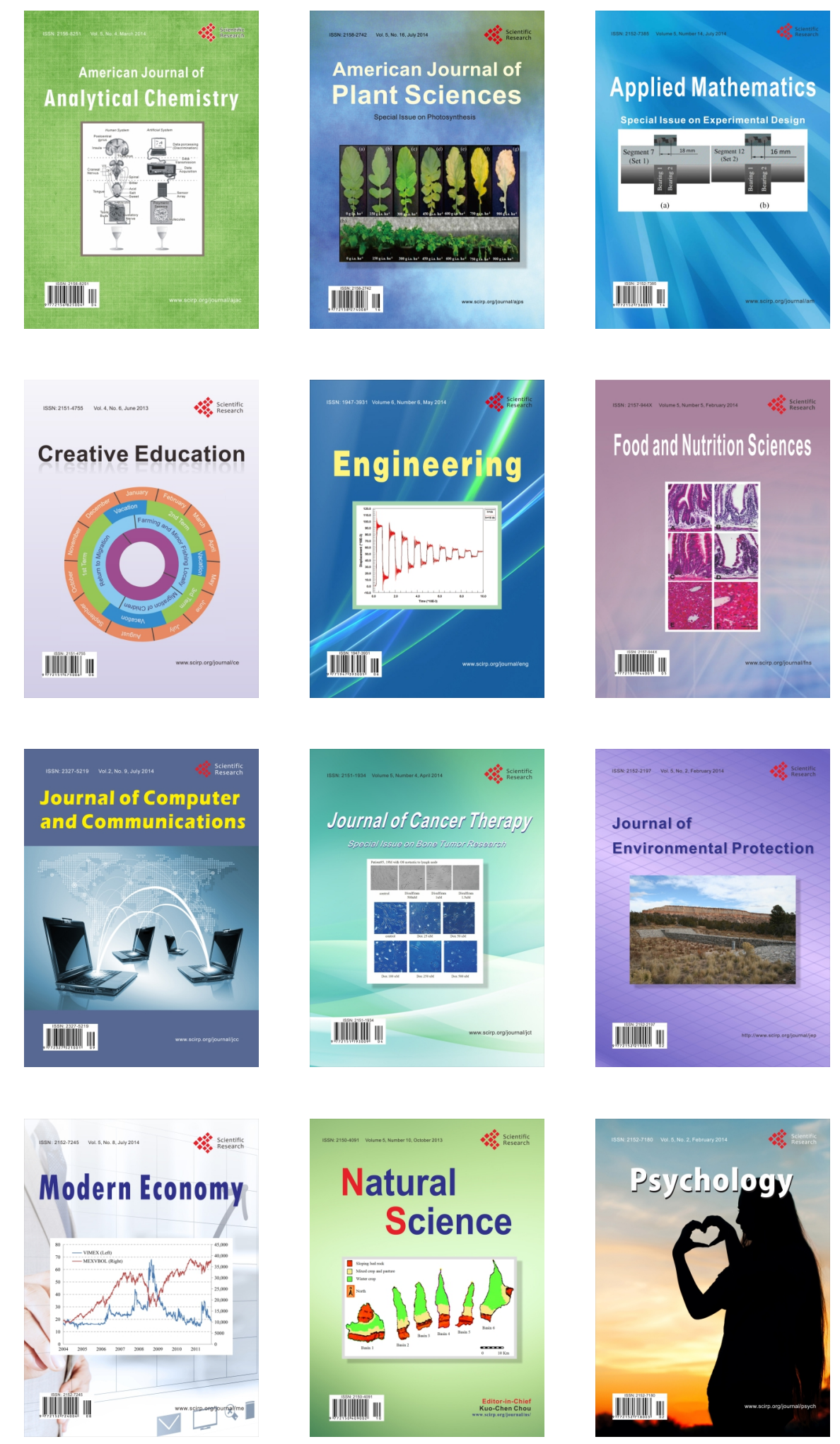